\title{
Amnion: a versatile tissue and cell source in tissue repair and regeneration
}

\author{
Simone Hennerbichler • Ornella Parolini
}

Received: 11 March 2014/Accepted: 20 March 2014/Published online: 27 April 2014

(C) Springer Science+Business Media Dordrecht 2014

Within the complex scenario of regenerative medicine and the sophisticated field of stem cell research and tissue engineering, it is interesting that biological waste as human placenta raises up and tells us its ancient application story and underlies that it is not essential only during the fetal development but also for future therapeutic application. Even more the amniotic membrane that constitutes the inner part of the amniotic sac has a long story of therapeutic applications.

This Special Issue of the journal Cell and Tissue Banking indeed is dedicated to this versatile tissue and collects some contributions dealing with amniotic membrane cells or rather the membrane itself. We span from the most well established use of the amniotic membrane in ophthalmology to the possible future use in regeneration of several tissues as well as to the understanding of the mechanisms of action of the cells derived from the amniotic membrane and to the envisaged possible use already for prenatal diseases. From all these evidences it is demanding to learn more about the characteristics and biological properties of the cells that constitutes the amniotic membrane. Furthermore, it is fundamental to identify the best strategies to better preserve and test this tissue and/or its derived cells for further clinical use.

Definitively, we still have to learn more about the properties of this versatile tissue and its cells and subpopulations, about their phenotype, their characteristics and potentiality; not to forget additional formal challenges which need to be met, like GMPcompliance as well as national and international requirements for tissues and ATMPs.

Of course the therapeutic potential still needs to be unraveled but we believe that in a few years amniotic membrane will be a very valuable, easy available source in regenerative medicine and tissue engineering leaving all ethical issues behind.

S. Hennerbichler $(\square)$

Red Cross Blood Transfusion Service of Upper Austria,

Linz, Austria

e-mail: simone.hennerbichler@o.roteskreuz.at

\section{S. Hennerbichler}

Austrian Cluster for Tissue Regeneration, Linz, Austria

O. Parolini

Centro di Ricerca E. Menni, Fondazione Poliambulanza-

Istituto Ospedaliero, Brescia, Italy

e-mail: ornella.parolini@poliambulanza.it 